\title{
Determination of bulk discharge current in the dielectric film of MEMS capacitive switches
}

\author{
M. S. Koutsoureli ${ }^{*}$ and G. J. Papaioannou \\ Solid State Physics Section, Physics Department, National and Kapodistrian University of Athens, \\ Panepistimioupolis, Zografos, Athens 15784, Greece.
}

\begin{abstract}
The present work presents a new method to calculate the discharge current in the bulk of dielectric films of MEMS capacitive switches. This method takes into account the real MEMS switch with non uniform trapped charge and air gap distributions. Assessment of switches with silicon nitride dielectric film shows that the discharge current transient seems to obey the stretched exponential law. The decay characteristics depend on the polarization field's polarity, a fact comes along with experimental results obtained from thermally stimulated depolarization currents (TSDC) method used in MIM capacitors.
\end{abstract}

\section{Introduction}

Capacitive RF MEMS switches are very promising devices for RF applications due to their small size, weight and possibility to be integrated in ICs. Despite these advantages reliability problems still hinder their commercialization. The most important problem is the dielectric charging, fact that causes erratic device behaviour [1,2]. So far the effects of dielectric charging in MEMS switches have been investigated by recording the shift of pull-in and pull-out voltages [2-5], as well as the voltage at which the pull-up capacitance becomes minimum [6]. The latter has been found to be thermally activated [7]. Moreover the charging processes have been investigated with the aid of MIM capacitors [8-10], by monitoring the decay of the top electrode potential [11]. The Kelvin Probe Force
Microscopy (KPFM) has recently been employed to investigate dielectric charging by monitoring the film surface potential [12-14].

The lifetime of a MEMS capacitive switch is directly related to the rates of injected charges during pull-down state and the collected charges in the bottom electrode during the pull-up state. Many attempts have been made in order to determine the charge decay rate in the dielectric film by monitoring the discharge currents in MIM capacitors. Both thermally stimulated depolarization current (TSDC) and discharge current transients (DCT) methods have shown [10] that in MIM capacitors the discharge current measured in the external circuit arises from collection of charges located near the injected electrodes. Recently it has been reported the measurement of the discharge current in MEMS capacitive switches [15], but the duration of

* Corresponding author: mkoutsoureli@phys.uoa.gr

Tel: +30 2107276722; Fax: +30 2107276711 
the current transient was limited to about $150 \mathrm{sec}$, thus providing little information about the parameters which determine the switch lifetime.

The aim of the present work is to demonstrate a new method that allows the determination of the discharge current in MEMS capacitive switches. Transients with duration in excess of $10^{3}$ sec have been monitored and current densities of the order of pico Ampere per unit area or even less have been calculated. The method takes into account the model of a real MEMS switch with non uniform trapped charge and air gap distributions [16]. The rate of the shift of bias for minimum pull-up capacitance and the dielectric film thickness allow the calculation of the bulk discharge current. The discharge current, determined by mechanisms such as hopping, percolation etc which are expected in an amorphous and disordered dielectric material, provides valuable information that can be used for further optimization and/or engineering of the dielectric material.

\section{Theoretical Background}

In order to determine the discharge current transient in a MEMS capacitive switch we adopt the device model proposed in [16] which includes a fixed nonflat metal plate of area $A$ covered with a dielectric film of uniform thickness $d_{\varepsilon}$, dielectric constant $\varepsilon_{r}$ and a volume charge density $\rho(x, y, z)$. Above it a rigid but nonflat moveable metal plate is fastened with a linear spring $k$ to a fixed wall above the dielectric layer at a rest position $d_{0}(x, y)$, as shown in Fig.1. According to [16] the electrostatic force $F_{e l}$ exerted to the top plate when a dc bias source of amplitude $V$ is applied to the two plates can be expressed in a compact form of:

$$
F_{e l}(\Delta)=\frac{A}{2 \varepsilon_{0}}\left[\left(V \mu_{\alpha}-\mu_{\beta}\right)^{2}+V^{2} \sigma_{\alpha}^{2}+\sigma_{\beta}^{2}-2 V \operatorname{cov}(\alpha, \beta)\right](1)
$$

where $\mu, \sigma^{2}$, and cov denote the mean, variance, and covariance, respectively, of the capacitance $\alpha(\mathrm{x}, \mathrm{y}, \Delta)$ and charge $\beta(x, y, \Delta)$ density distributions. The distributions of capacitance per unit area and charge density induced at armature area are respectively:

$$
\begin{aligned}
& \alpha(x, y, \Delta)=\frac{\varepsilon_{0}}{\left[d_{0}(x, y)-\Delta\right]-\left(d_{\varepsilon} / \varepsilon_{r}\right)} \\
& \beta(x, y, \Delta)=\frac{d_{\varepsilon}}{\varepsilon_{r} \varepsilon_{0}} \cdot \psi_{e q}(x, y) \cdot \alpha(x, y)
\end{aligned}
$$

$\Delta$ denotes the displacement of the moving electrode from its rest position and $\psi_{e q}(x, y)$ is the equivalent surface charge density distribution.

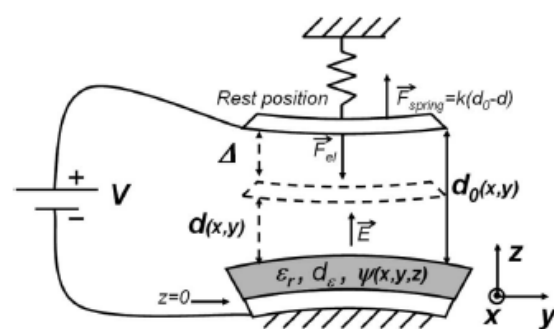

Figure 1: Model of a capacitive switch with non uniform trapped charge and air gap distributions [16].

In the general case of distributed equivalent charge $[\psi(x, y, z)]$ and air gap $\left[d_{0}(x, y)\right]$, Eq. 1 cannot be further simplified. The bias at which the up-state capacitance attains minimum $\left(V_{m}\right)$ is the bias for which the electrostatic force becomes minimum independently of the charge and air gap distributions and it is given by:

$$
V_{m}=\frac{\mu_{\alpha} \mu_{\beta}+\operatorname{cov}(\alpha, \beta)}{\mu_{\alpha}^{2}+\sigma_{\alpha}^{2}}
$$

If for the sake of simplicity it is assumed that the capacitance variance is low, then Eq. 3 simplifies to:

$$
V_{m}=\frac{\mu_{\beta}}{\mu_{\alpha}}=\frac{d_{\varepsilon}}{\varepsilon_{r} \varepsilon_{0}} \mu_{\psi}
$$

where $\mu_{\psi}$ represents the mean value of $\psi_{e q}(x, y)$.

According to these the net discharge current density is given by:

$$
J_{\text {disch }}(t)=-\frac{d \mu_{\psi}(t)}{d t}=-\frac{\varepsilon_{r} \varepsilon_{0}}{d_{\varepsilon}} \cdot \frac{d V_{m}(t)}{d t}
$$

Here it must be pointed out that Eq.5 describes the average net charge discharge current density. Therefore in a switch where the net charge is zero Eq.5 will lead to a zero current.

\section{Experimental approach}

The switches used in the present work were fabricated with a standard photolithographic process on high resistivity silicon wafers on top of which $\mathrm{SiO}_{2}$ film was deposited. The dielectric film is PECVD $\mathrm{Si}_{3} \mathrm{~N}_{4}$ deposited at $300^{\circ} \mathrm{C}$. The thickness of the dielectric film is $250 \mathrm{~nm}$. The membrane is an evaporated titaniumgold seed layer electroplated to a thickness of $2 \mu \mathrm{m}$. Under no applied force, the membrane is normally suspended about $2.5 \mu \mathrm{m}$ above the dielectric. The sacrificial layer was removed with resist stripper and the switches were dried using a critical point dryer. The active area of the switches was about $2.5 \cdot 10^{-5} \mathrm{~cm}^{2}$.

The pull-up capacitance voltage (C-V) characteristics were monitored with a Boonton 72B capacitance meter while sweeping the voltage in $50 \mathrm{mV}$ 
steps. The pull-in voltage of the switches was $20 \mathrm{~V}$. The assessment was carried out for two switches after being stressed for $5 \mathrm{~min}$ at $-30 \mathrm{~V}$ and $+30 \mathrm{~V}$. In all cases the discharge process was monitored for $14000 \mathrm{sec}$. The bias for pull-up state capacitance minimum was determined by fitting a parabola to the experimental data. Finally, in the present work for the sake of simplicity it was assumed that the capacitance variance is very small, in order to satisfy the conditions to apply Eq.5.

\section{Results and discussion}

The shift of pull-up state capacitance voltage $(\mathrm{C}-\mathrm{V})$ characteristics for a switch after being stressed with $-30 \mathrm{~V}$ for $5 \mathrm{~min}$ is presented in Fig.2. For both stress bias polarities the absolute value of the bias $\mathrm{V}_{\mathrm{m}}$ at which the pull-up capacitance attains its minimum decreases with time, as presented in Fig.3, while the minimum pull-up capacitance gradually increases with time after stress, fact that can be attributed to creep and/or non-uniform charging [6] since the stress was unipolar.

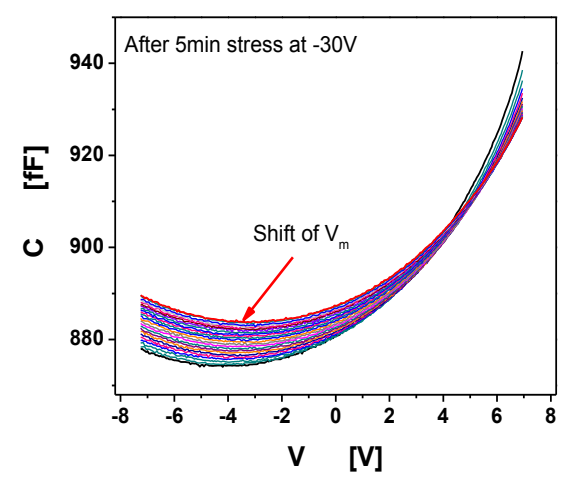

Figure 2: Evolution of pull-up state capacitance-voltage characteristic and the shift of capacitance minimum.

Taking into account that the dielectric film in the utilized switches is amorphous silicon nitride it is expected that the charge decay, arising from dipole and space charge polarization mechanisms, will obey a stretched exponential law of the form:

$$
V_{m}=V_{0} \cdot \exp \left[-\left(\frac{t}{\tau}\right)^{\beta}\right]
$$

The fitting revealed significant differences in time constants for the two stress bias polarities while the stretched factor differs slightly for the two cases. More specifically, for the case of stress bias of $-30 \mathrm{~V}$ the stretched factor is $\beta=0.31$ and the time constant is found to be $\tau=1.3 \cdot 10^{5} \mathrm{~s}$, whereas for the case of $+30 \mathrm{~V}$ the stretched factor is $\beta=0.39$ and the time constant is $\tau=3.2 \cdot 10^{2} \mathrm{~s}$. The value of the stretched factor $\beta$ for both cases reveals a complex process. The difference in time constants may be attributed to differences in injected charge mechanisms related to differences of the dielectric film near the bottom and the top plate.

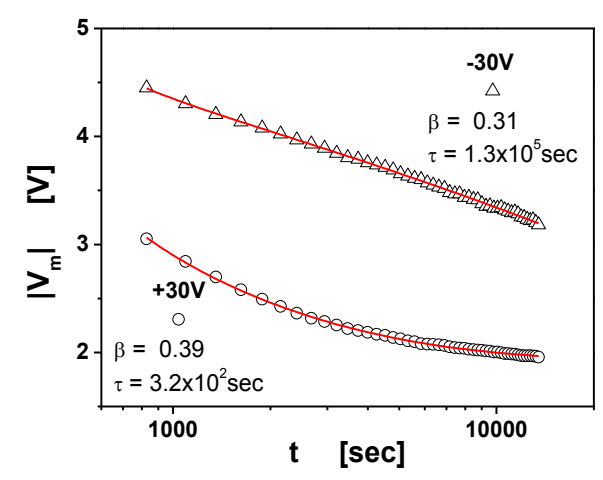

Figure 3: The shift of bias $\mathrm{V}_{\mathrm{m}}$ with time of two switches after being stressed for $5 \mathrm{~min}$ at $+30 \mathrm{~V}$ and $-30 \mathrm{~V}$.

The discharge current density, according to Eq.6 obtains the form:

$$
J_{\text {disch }}(t)=\mu_{\psi, 0} \cdot\left(\frac{\beta}{\tau}\right) \cdot\left(\frac{t}{\tau}\right)^{\beta-1} \cdot \exp \left[-\left(\frac{t}{\tau}\right)^{\beta}\right]
$$

where $\mu_{\psi, 0}$ refers to the initial net charge. In Fig.4 the discharge current transients for both stress bias polarities is presented.

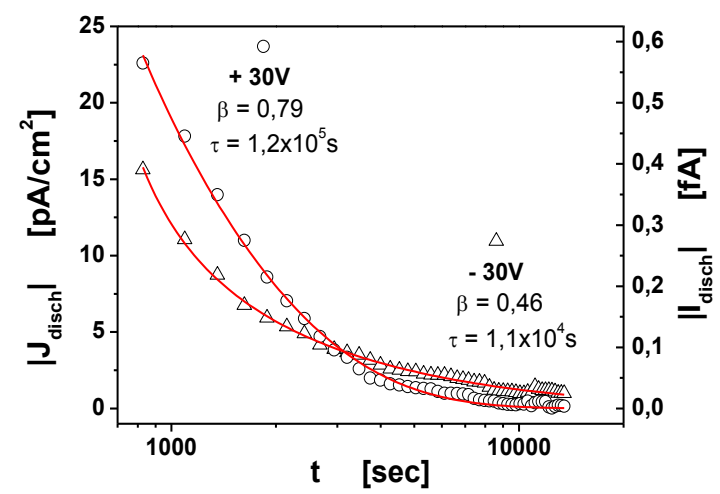

Figure 4: Bulk discharge current calculated for two switches, after being stressed for $5 \mathrm{~min}$ at $-30 \mathrm{~V}$ and $+30 \mathrm{~V}$.

The discharge current is found to be greater for the case of positive stress bias and the parameters $\tau$ and $\beta$ obtained from fitting the experimental results with Eq.7 revealed differences for the two bias polarities. In case of negative stress bias it was found that $\beta=0.46$ and 
$\tau=1.1 \cdot 10^{4} \mathrm{~s}$, while $\beta=0.79$ and $\tau=1.2 \cdot 10^{5} \mathrm{~s}$ in case of $+30 \mathrm{~V}$ stressing bias.

Moreover, the parameters $\beta$ and $\tau$ obtained for $\mathrm{V}_{\mathrm{m}}$ differ from those obtained for $\mathrm{J}_{\text {disch }}$, fact that may be attributed to the fact that $\mathrm{V}_{\mathrm{m}}$ and $\mathrm{J}_{\text {disch }}$ are determined from different contributing mechanisms in the time window of the experiment. The use of longer time window of observation will give a better insight to these differences.

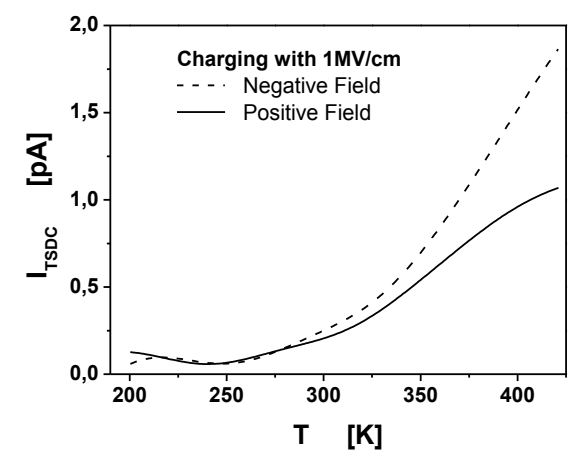

Figure 5: Depolarization current measured in a TSDC experiment. The polarity of the field refers to the polarity of the top electrode of MIM capacitor.

Finally, the asymmetry of discharge current found for the two different stress bias polarities comes along with the asymmetry obtained from TSDC assessment in MIM capacitors with same characteristics as MEMS capacitive switches, as shown in Fig.5. This asymmetry is related to the fact that carrier transport via silicon dangling bond defect states in SiN amorphous films occurs due to the motion of holes and hole transport is favored over electron transport [17].

In conclusion, it has been presented a new method to calculate the discharge current in the bulk of the dielectric film of MEMS capacitive switches. The shift of the bias for the minimum of the pull-up capacitance and the dielectric film thickness are used to calculate current densities in the order of pico Ampere per unit area, a value that is much lower than any reported one. The bulk discharge current has been found to obey the stretched exponential law and its parameters depend on the polarity of the polarized field. Greater bulk discharge current with greater time constant has been found in case of positive stress bias. The knowledge of the magnitude and time dependence of the bulk discharge current provides valuable information on the further optimization and/or engineering of the MEMS dielectric films.

\section{References}

[1] J. Wibbeler, G. Pfeifer and M. Hietschold, Sensors and Actuators A 71 (1998) 74-80.

[2] W. M. van Spengen, R. Puers, R. Mertens and I. de Wolf, J. Micromech. Microeng. 14 (2004) 514-521.

[3] S. Melle, D. De Conto, L. Mazenq, D. Dubuc, B. Poussard, C. Bordas, K. Grenier, L. Bary, O. Vendier, J. L. Muraro, J. L. Cazaux and R. Plana, IEEE Tans. on Microwave Theory and Techniques, Microelectronics Reliability 45 (2005) 1770-1775.

[4] Z. Peng, X. Yuan, J. C. M. Hwang, D. I. Forehand and C. L. Goldsmith, International Microwave Symposium (2007) 1817-1820.

[5] D. Mardivirin, D. Bouyge, A. Crunteanu, A. Pothier and P. Blondy, International Microwave Symposium (2008) 33-36.

[6] R.W. Herfst, H.G.A. Huizing, P.G. Steeneken, and J. Schmitz, IEEE 45th Annual International Reliability Physics Symposium (2007) 417-420.

[7] G. Papaioannou, J. Papapolymerou, P. Pons and R. Plana, Applied Physics Letters 90 (2007) 233507.

[8] X. Yuan, J. C. M. Hwang, D. Forehand and C. L. Goldsmith, International Microwave Symposium (2005) 753-756.

[9] E. Papandreou, M. Lamhamdi, C. M. Skoulikidou, P. Pons, G. Papaioannou and R. Plana, Microelectronics Reliability 47 (2007) 1812-1817.

[10] M. Lamhamdi, P. Pons, U. Zaghloul, L. Boudou, F. Coccetti, J. Guastavino, Y. Segui, G. Papaioannou, R. Plana, Microelectronics Reliability 48 (2008) 12481252.

[11] U. Zaghloul, M. Koutsoureli, H. Wang, F. Coccetti, G. Papaioannou, P. Pons and R. Plana, Microelectronics Reliability 50 (2010) 1615-1620.

[12] R. W. Herfst, P. G. Steeneken, J. Schmitz, A. J. G. Mank and M. van Gils, 46th Annual International Reliability Physics Symposium (2008) 492-495.

[13] U. Zaghloul, F. Coccetti, G. Papaioannou, P. Pons and R. Plana, IEEE International Reliability Physics Symposium (IRPS) (2010) 237-245.

[14] U. Zaghloul, B. Bhushan, P. Pons, G. J. Papaioannou, F. Coccetti and R. Plana, Nanotechnology 22 (2011) 035705.

[15] D. Molinero and L. Castaner, Applied Physics Letters 94 (2009) 043503.

[16] X. Rottenberg, I. De Wolf, B. K. J. C. Nauwelaers, W. De Raedt and H. A. C. Tilmans, J. of Microelectromechanical Systems, vol. 16, No. 5, (2007) 1243-1253.

[17] J. M. Shanon and B. A. Morgan, J. of Applied Physics 86, No3 (1999) 1548-1551.

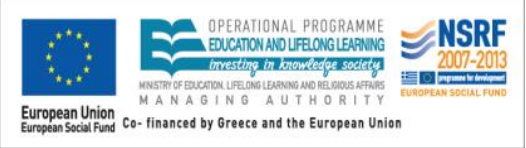

